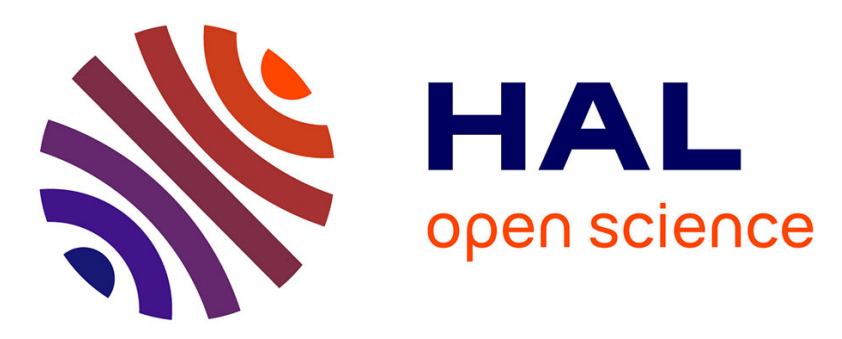

\title{
Application of Growth Curve in Agricultural Scientific Research
}

\author{
Zeng-Hui Wang, Yan-Jun Zhao, Yang Liu, Dong-Yan Huang
}

\section{To cite this version:}

Zeng-Hui Wang, Yan-Jun Zhao, Yang Liu, Dong-Yan Huang. Application of Growth Curve in Agricultural Scientific Research. 11th International Conference on Computer and Computing Technologies in Agriculture (CCTA), Aug 2017, Jilin, China. pp.285-292, 10.1007/978-3-030-06137-1_26 . hal02128302

\section{HAL Id: hal-02128302 \\ https://hal.inria.fr/hal-02128302}

Submitted on 14 May 2019

HAL is a multi-disciplinary open access archive for the deposit and dissemination of scientific research documents, whether they are published or not. The documents may come from teaching and research institutions in France or abroad, or from public or private research centers.
L'archive ouverte pluridisciplinaire HAL, est destinée au dépôt et à la diffusion de documents scientifiques de niveau recherche, publiés ou non, émanant des établissements d'enseignement et de recherche français ou étrangers, des laboratoires publics ou privés.

\section{(c)(1)}

Distributed under a Creative Commons Attribution| 4.0 International License 


\title{
Application of Growth Curve in Agricultural Scientific Research
}

\author{
Zeng-hui Wang ${ }^{1}$, Yan-jun Zhao ${ }^{2(\bowtie)}$,yang Liu ${ }^{1}$, Dong-yan Huang ${ }^{2}$ \\ ${ }^{1}$ College of Humanities \& Sciences of Northeast Normal University, \\ Changchun ,China \\ ${ }^{2}$ Key Engineering Bionics Laboratory of Jilin University Education Ministry
}

\begin{abstract}
This paper introduces the application of logistic curve in agricultural science, and gives a division method of parameter estimation of logistic curve. Because the logistic curve contains three parameters, it is not possible to use least square method to estimate the parameters directly. In this paper, the parameter $\mathrm{C}$ is estimated by the three (four) point method at first. Then the two steps method is used to estimate the parameters b and a respectively. Through a practical example with the new method, the example shows that the precision of this method is relatively high..
\end{abstract}

Key words: Logistic curve; Parameter estimation; division method;

\section{Introduction}

The logistic growth curve is the most famous curve among the building-up curve, which was originally developed in the study of population growth, and later P.F.Ver hulst , a mathematician in Belgium has summed up it as a mathematical model.

$$
y(t)=\frac{c}{1+a e^{-b t}}(a>0, b>0, c>0)
$$

The parameter $\mathrm{t}$ represents time, the parameter $\mathrm{y}$ is the increase in the $\mathrm{t}$ times.

The characteristic of this kind of curve is that when the value of the $t$ is small, the value of the y grows slowly. As time goes on, y changes into blocks, when the parameter $t$ gets the critical value, and the increase of the value of $\mathrm{Y}$ begins to slow down again. Finally, the value of $\mathrm{Y}$ is close to a certain value.According to this characteristic, logistic growth curve is widely applied in life science research.For example, the logistic curve is used to describe the growth process of plant height, leaf area, the growth of the height and weight of the young in the study of juveniles. Especially, the accumulation of starch, fat and protein in food crops is consistent with the logistic curve. Therefore, it is very meaningful to study the parameter estimation method of logistic curve.

The ordinary least square method and the Marquardr method can estimate the parameters in the logistic growth curve, but the accuracy of ordinary least squares method is low, and the Marquardr method is an iterative method for technicians, for the calculation is more complex. A new approach in logistic growth curve of parameter estimation is given in this paper.

Fund project: national key research and development project funded by National Science and Technology department (project number: 2016YFD0700300)

Author profile: Wang Zeng hui (1956 -), male, research direction: statistics and optimization method;

Corresponding author: Yan-Jun Zhao (1979 -), male, research direction: partial differential equation and its 
application, the application of time series analysis and data mining, E-mail:yjzchsnenu@163.com

\section{The general estimation approach in logistic growth curve}

\subsection{Estimation of the parameter $\mathrm{C}$}

Assuming that some actual measured data as $\quad\left(t_{i}, y_{i}\right)(i=1,2, \cdots, n)$. Make the value of $t_{i}$ is spaced equally, we have $t_{i}=t_{1}+(i-1) h,(i=1,2, \cdots, n)$.

If $\mathrm{n}$ is odd, select the initial point, midpoint and terminal point. In this case, a formula as follows:

$$
\hat{c}=\frac{y_{\frac{n+1}{2}}^{2}\left(y_{1}+y_{n}\right)-2 y_{1} y_{\frac{n+1}{2}} y_{n}}{y_{\frac{n+1}{2}}^{2}-y_{1} y_{n}}
$$

If $\mathrm{n}$ is even number,select the $\left.\left(t_{1}, y_{1}\right), \underset{\frac{n}{2}}{\left(t_{2}\right.}, y_{\frac{n}{2}}\right), \underset{\frac{t_{n}+1}{2}}{\left(t_{\frac{n}{2}+1}\right.}, y_{n},\left(t_{n}, y_{n}\right)$. In this case, a formula as follows:

$$
\hat{c}=\frac{\left(y_{1}+y_{n}\right) y_{\frac{n}{2}} y_{\frac{n}{2}+1}-y_{1} y_{n}\left(y_{\frac{n}{2}}+y_{\frac{n}{2}+1}\right)}{y_{\frac{n}{2}} y_{\frac{n}{2}+1}-y_{1} y_{n}}=\frac{y_{1} y_{n}\left(y_{\frac{n}{2}}+y_{\frac{n}{2}+1}\right)-y_{\frac{n}{2}} y_{\frac{n}{2}+1}\left(y_{1}+y_{n}\right)}{y_{1} y_{n}-y_{\frac{n}{2}} y_{\frac{n}{2}+1}}
$$

\subsection{Estimation of parameter $a$ and $b$}

When the parameter $\mathrm{C}$ is estimated by using the above formula, there are only two parameters to be estimated in the model 1.The least squares method can be used to solve it.

First of all, the type (1) needs to be became the reciprocal value, and then take the natural logarithm.

$$
\ln \frac{c-y}{y}=\ln a-b t
$$

We use the following notation: $\mathrm{Y}=\ln \frac{c-y}{y}, A=\ln a, B=-b$

The above formula can be transformed into a linear model.

$$
Y=A+B t
$$

$$
\left\{\begin{array}{l}
\hat{A}=\bar{Y}-\hat{B} \bar{t} \\
\hat{B}=\frac{\sum_{i=1}^{n} t_{i} Y_{i}-\frac{1}{n}\left(\sum_{i=1}^{n} t_{i}\right)\left(\sum_{i=1}^{n} Y_{i}\right)}{\sum_{i=1}^{n} t_{i}^{2}-\frac{1}{n}\left(\sum_{i=1}^{n} t_{i}\right)^{2}}=\frac{\sum_{i=1}^{n} t_{i} Y_{i}-n \bar{t} \bar{Y}}{\sum_{i=1}^{n} t_{i}^{2}-n \bar{t}^{2}}
\end{array}\right.
$$

Among, $\bar{Y}=\frac{1}{n} \sum_{i=1}^{n} Y_{i}, \bar{t}=\frac{1}{n} \sum_{i=1}^{n} t_{i} \quad \hat{a}=e^{\hat{A}}, \hat{b}=-\hat{B}$.

The estimation of the parameter A and the parameter B is calculated by the formula (5), we have $\hat{a}=e^{\hat{A}}$, $\hat{b}=-\hat{B}$, The three parameters of the model (1) are estimated.

\section{A new approach in logistic growth curve of parameter estimation}

Let's say that the logistic growth curve is the following formula

$$
y=\frac{c}{1+a e^{-b t}}
$$


$a>0, b>0, c>0$ (The estimated parameters)

In the case of the estimation of the pending parameters in model(1), we take three steps.

\subsection{Estimation of parameter $\mathrm{c}$}

The estimation of parameter c is still used in the three points or four points method, as the formula (2) or (3).

\subsection{Estimation of parameter $b$}

A set of measured data is $\left(\mathrm{t}_{i}, y_{i}\right), i=0,1,2, \cdots, n$. The formula for the parameter $\mathrm{b}$ is derived. Take the inverse of the (1) and transform it into the following model:

$$
\frac{c-y}{y}=a e^{-b t}
$$

$Y=\frac{c-y}{y}$, The upper model is changed into the exponential model

$$
\mathrm{Y}=a e^{-b t}
$$

$\hat{Y}_{i}=a e^{-b t_{i}}, \hat{Y}_{i-1}=a e^{-b t_{i-1}}, \quad i=2,3, \cdots, n, Y_{i}=\frac{c-y_{i}}{y_{i}}, i=1,2, \cdots, n$.

Eliminate the parameter a in the (6) model,therefore

$$
\frac{\hat{Y}_{i}}{\hat{Y}_{i-1}}=\frac{a e^{-b t_{i}}}{a e^{-b t_{i-1}}}=e^{-b\left(t_{i}-t_{i-1}\right)}
$$

The (7) formula only has one parameter b which needs to be estimated. Now we use the least square method to estimate the parameter b.

Get the natural log for the(7) formula

$$
\ln \frac{\hat{Y}_{i}}{\hat{Y}_{i-1}}=-b\left(t_{i}-t_{i-1}\right)
$$

Thus we set $x_{i}=\ln \frac{Y_{i}}{Y_{i-1}}=\ln Y_{i}-\ln Y_{i-1}, \hat{x_{i}}=\ln \frac{\hat{Y_{i}}}{\hat{Y_{i-1}}}=\ln \hat{Y}-\ln \hat{Y_{i-1}}=-b\left(t_{i}-t_{i-1}\right)$,

$\Delta t_{i}=t_{i}-t_{i-1}, i=2,3, \cdots, n$.So the formula (8) becomes the following model.

$$
\hat{x}_{i}=-b \Delta t_{i}
$$

By the least squares principle we have

$$
Q(b)=\sum_{i=1}^{n}\left(x_{i}-\hat{x}_{i}\right)^{2}=\sum_{i=2}^{n}\left(x_{i}+b \Delta t_{i}\right)^{2}=\min
$$

Use the principle of the extremum of the function

$$
\frac{d Q(b)}{d b}=2 \sum_{i=2}^{n}\left(x_{i}+b \Delta t_{i}\right) \Delta t_{i}=0
$$

So we have a estimated model about parameter b 


$$
\hat{b}=\frac{-\sum_{i=2}^{n} x_{i} \Delta t_{i}}{\sum_{i=2}^{n} \Delta t_{i}^{2}}=-\frac{\sum_{i=2}^{n} \ln \left(\ln \frac{c-y_{i}}{c-y_{i-1}} \cdot \frac{y_{i-1}}{y_{i}}\right) \Delta t_{i}}{\sum_{i=2}^{n} \Delta t_{i}^{2}}
$$

\subsection{Estimation of parameter a}

Exponential model (6) of the parameters is given by (10), so only the parameter in the model (6) can be estimated, here we still using the least squares estimation, we note $\hat{Y}_{i}=a e^{b t_{i}}$. The parameter a should satisfy the following model gains the minimum.

$$
Q(a)=\sum_{i=1}^{n}\left(Y_{i}-\hat{Y}_{i}\right)^{2}=\sum_{i=1}^{n}\left(Y_{i}-a e^{-b t_{i}}\right)^{2}
$$

Use the principle of the extremum of the function.

$$
\frac{d Q(a)}{d a}=-2 \sum_{i=1}^{n}\left(Y_{i}-a e^{-b t_{i}}\right) e^{-b t_{i}}=0
$$

So we get the estimated formula of parameter a,

$$
\hat{a}=\frac{\sum_{i=1}^{n} Y_{i} e^{-b t_{i}}}{\sum_{i=1}^{n} e^{-2 b t_{i}}}=\frac{\sum_{i=1}^{n} \frac{c-y_{i}}{y_{i}} e^{-b t_{i}}}{\sum_{i=1}^{n} e^{-2 b t_{i}}}
$$

\section{The logistic growth curve is fitted to the optimum test.}

In general, the equation of logistic growth curve regression equation is measured by the correlation index $\left(R^{2}\right)$ and the residual sum of the sum of the squares of residuals( $S e$ ), which the definitions are as follows,

$$
\mathrm{R}^{2}=1-\frac{\sum_{i=1}^{n}\left(y_{i}-\hat{y}_{i}\right)^{2}}{\sum_{i=1}^{n}\left(y_{i}-\bar{y}\right)^{2}} \quad S e=\sum_{i=1}^{n}\left(y_{i}-\hat{y}_{i}\right)^{2}
$$

\section{Case analysis}

\subsection{Model application}

We study the pattern of the weight of the chicken.The silky fowl come from Xichuan county in Henan province, is one of the excellent local breeds in Henan province, it has the high protein content, amino acid, a variety of vitamins and trace elements as well, while the cholesterol content is low, people enjoy it at the table.In order to get profit, it is meaningful to understand its growth rule. Table 1 shows the growth observation data of the silky fowl.

Table 1

\begin{tabular}{cccccccccc}
\hline $\mathrm{t}$ (yearling) & 1994 & 2 & 4 & 6 & 8 & 10 & 12 & 14 & 16 \\
\hline$y_{i}$ (weight) & 30.58 & 82.45 & 172.28 & 312.27 & 434.90 & 589.70 & 759.25 & 921.93 & 1026.93 \\
$\hat{y}_{i}$ (weight) & 33 & 64.31 & 121.66 & 220.1 & 370.29 & 563.6 & 761 & 957.2 & 1037.7 \\
\hline
\end{tabular}

(weight: g)

Now we use the logistic growth curve to fit the pattern of the growth of the chicken. 


$$
y=\frac{c}{1+a e^{-b t}}
$$

Take $t=0$, due to a total of nine data, namely, $n=9$, therefore the three point method can be used to estimate the parameter $c$ in the logistic growth curve.

Get the dates for 30.58, 434.9, 1026.93 into type(2), we have,

$$
\hat{c}=\frac{30.58 \times 434.9 \times 1026.93-434.9 \times(30.58+1026.93)}{30.58 \times 1026.93-434.9 \times 434.9}=1181.466
$$

The data in table 1 is evaluated in the formula (10),the parameter b can be estimated,

$$
\hat{b}=-\frac{\sum_{i=2}^{9} \ln \left(\ln \frac{c-y_{i}}{c-y_{i-1}} \cdot \frac{y_{i-1}}{y_{i}}\right) \Delta t_{i}}{\sum_{i=2}^{9} \Delta t_{i}^{2}}=0.34508
$$

The parameter a can be calculated.

$$
\hat{a}=\frac{\sum_{i=1}^{9} \frac{c-y_{i}}{y_{i}} e^{-b t_{i}}}{\sum_{i=1}^{9} e^{-2 b t_{i}}}=34.6398
$$

So we have the logistic growth curve model of the pattern of growth of the silky fowl.

$$
y=\frac{1181.4661}{1+34.6398 e^{-0.3451 t}}
$$

The accuracy of the model (14) needs to be thought.We count the fitting value for the first time.

$$
\hat{y}_{i}=\frac{1181.4661}{1+34.6398 e^{-0.3451 t_{i}}} \quad(i=1,2, \ldots, 9)
$$

Counted fitting values are listed in table 1 , from the data in table 1 in the type (13) we conclude the correlation index, thus it can be seen that the fitting precision of the curve is relatively high.

\subsection{Conclusion}

The logistic model (14) was applied to study the weight growth rule of the silky fowl in Zhejiang province.

Take the derivative of the formula (14), we have

$$
y^{\prime}(t)=14123.476 e^{-0.3451 t}\left(1+34.6398 e^{-0.3451 t}\right)^{2}
$$

(1)The value of first derivative in the time of $t$ is the reflection of the growing speed of the silky fowl in Zhejiang province.

If $t_{1}<t_{2}$, we have $y^{\prime}\left(t_{1}\right)<y^{\prime}\left(t_{2}\right)$.It is shown that the weight gain of the silky fowl in Zhejiang province at the time of $t_{2}$ is faster than the time of $t_{1}$.If $t_{1}<t_{2}$, we have $y^{\prime}\left(t_{1}\right)>y^{\prime}\left(t_{2}\right)$.It is shown that the weight gain of the silky fowl in Zhejiang province at the time of $t_{1}$ is faster than the time of $t_{2}$.

(2)Take the first derivative with respect to the formula (15) again, set $y^{\prime \prime}=0$, we can find the inflection point of the curve, and the inflection point corresponds with the time point is 11.5. We know the speed of the second derivative reaction of chicken weight growth.

When $y>0$, it indicates that the chicken's weight gain is in the fast growth period. When $y^{\prime \prime}<0$, it 
indicates that the weight gain of chickens enters the deceleration period.

When $t<12$ (yearling), the weight is in the fast growth period. When the time of $t>12$ (yearling), although the weight is still growing, but it has been in the growing period with low speed.

\section{Reference}

[1]Tang Wu-Xiang.A new method of estimation of the GM(1,1) Model Parameters and Hypothesis Tsting.Systems Engineering-Theory \& Practice,1995,15(3):20-25.[in Chinese]

[2]Zhao Hong,Wang Zeng-hui.New method of logistic model parameter estimation and application. Journal of Quantitative Economics, 2014,Volume 31(3): 103--105.[in Chinese]

[3]Fan Guo-bing,An estimation of the method and application of the logistic model parameter.Journal of Quantitative Economics, 2010,27(1):105-110. [in Chinese]

[4]Cheng Mao.Lin.ExtenSion and application of the logistic growth curve.Operations Research and Management Science, 2003,12(3):85-88[(in Chinese)

[5]Yin Zuo-yun.Study of logistic growth curve fitting method, Journal of Applied Statistics and Management, 2002,21(1):41-46.[in Chinese]

[6]Zhao Yi-bin,Hu Shun-tian,Zhao Yong-an.General form and new construction method of growth curve in mathematical model.Journal of Institute of Disaster Prevention,2003(03):11-16.[in Chinese]

[7]D.A.Ratkowsky.A practical method for the unification of nonlinear regression models[D]. Nanjing University Press,1986.

[8]Yang Yi-Qun,Wu Liang-huan, Zhang Huo-fa.Comparison of Gompertz curve and Logistic growth curve.Journal of biological mathematics,1996(03):166-168.[in Chinese]

[9]Chang Zhen-hai,Liu wei.Application in Logistic regression model.Journal of Yanbian University(Natural Science Edition),2012,38(01):28-32.[in Chinese]

[10]Yu Ai-hua.Research on Logistic model.Nanjing forestry university,2003.[in Chinese]

[11]Xu liang, Meng Xian-min,Su Gui-mei.Application of animal growth curve model to analyze the growth and development of larva of different tussah silkworm varieties Science of Sericulture, 2013,39(03):620-623.[in Chinese]

[12]Theory of the growth of silkworm larvae and its application. Ueda S. JARQ Japan Agricultural Research Quarterly. 1982.

[13]Mignon-Grasteau S.Genetic parameters of growth curve parameters in male and female chickens, British poultry science, 1999 , Vol.40 (1), pp.44-5. 